\title{
Editorials
}

\section{Understanding and managing dental and orofacial pain in general practice}

Pain in the trigeminal system, including dental pain, is 'special'. Not only is the trigeminal nerve the largest sensory nerve in the body, represented by over $50 \%$ of the sensory cortex, but it is the only sensory nerve with an intracranial distal root ganglion: the trigeminal ganglion. It is also the 'great protector' of many vital structures, including the meninges, eyes, ears, nose, and mouth, underpinning survival and enjoyment of life. As such, any pain within the trigeminal system is met with varying degrees of angst, fear, and trepidation. This explains, in part, why phobia of dentists and dental procedures is so common. In addition, chronic pain within the trigeminal system has a significant negative quality-of-life impact, including suicidal tendencies in some patients.

Healthy (nociceptive and inflammatory) and unhealthy (neuropathic and dysfunctional) pains in the trigeminal system tend to be interpreted and reacted to in many different ways under different circumstances. ${ }^{2}$

Dental pain is unique, given that pulpal nerves experience pain only on stimulation. Thus, the teeth are the only parts of the body within which allodynia is 'normal'. Dental pain is amongst the most common forms of pain known to humankind, elicited typically by dental caries, resulting in inflammation of the dental pulp (Figure 1). Initially, symptoms are temporary acute allodynic pain (lasting seconds) well localised to the affected tooth, elicited by hot and cold stimuli and sugary and acidic foods (reversible pulpitis); subsequently, there is spontaneous, acute allodynic pain of variable duration (minutes), possibly poorly localised to the affected tooth (irreversible pulpitis). The treatment for reversible pulpitis is to manage dental decay and restore the tooth with a restoration; for irreversible pulpitis it is to remove the decay and the dental pulp, and place a restoration following endodontic treatment (root filling), or extract the tooth.

Pulpitis is caused also by trauma to the teeth exposing dentine and possibly the dental pulp (www.dentaltrauma.co.uk), gingival recession, and severe tooth wear. Exposed dentine may be exquisitely sensitive, eliciting allodynia or nociceptive pain. If pulpal inflammation goes untreated, increased vascularity within the pulp space 'self-strangulates' the pulp. This process may extend over a day or two, typically causing severe, unrelenting allodynic pain in the region of the affected tooth. The pulp finally becomes non-vital, changing the pain presentation. A non-vital tooth may remain non-symptomatic; however, when bacteria, or bacterial toxins, pass through the apex of the tooth into the jaw inflammation occurs - periapical periodontitis. The symptoms, spontaneous toothache or pain on biting on the tooth, are regionally localised. The treatment is to remove decay and the remnants of the dead dental pulp and place a restoration following endodontic treatment, or extract the tooth. If untreated a periapical abscess develops, typically causing severe symptoms (spontaneous regional pain). The treatment is to remove decay, complete endodontic treatment, including management of the periapical abscess, and place a restoration, or extract the tooth.

If treatment is delayed and the abscess spreads to local tissue spaces (sublingual, submandibular, masseteric, buccal, infratemporal, and parapharyngeal) then the patient will present with marked facial swelling. Drainage and extraction of the tooth causing the infection are normally possible under local anaesthesia. If the abscess does not drain through the tooth socket, drainage can be achieved by opening the affected tissue space (s). If pus drainage is limited and/or the patient displays signs of systemic infection (pyrexia, malaise), respiratory difficulty, or dysphagia, then URGENT treatment is required. This typically involves extraction of the tooth and drainage, supported with parenteral antibiotics and admission for respiratory observation and support.

In managing dental pain the indications for prescribing antibiotics are very rare the cause of the pain being typically pulpal inflammation and possibly localised infection best treated by operative (dental) intervention. In contrast, analgesics, reassurance, and access to dental care are essential. Inappropriate prescription of antibiotics may complicate and compromise, rather than aid, the management of dental pain.

A 'toothache' type pain can be caused by inflammation of the gum surrounding a erupting tooth. Such pain may be experienced during teething and when both deciduous and permanent teeth erupt in infants and children. At 18-22 years of age, third permanent molar ('wisdom') teeth may try to erupt with limited space available, resulting in pericoronitis linflammation around the crown), an indication for surgical removal of the tooth. Pain caused by biting down on the inflamed tissue is best managed by reduction or extraction of the opposing tooth.

Dental and postoperative/surgical dental pain are best managed using routine oral analgesics. The optimum analgesia for adults is a combination of ibuprofen (ideally $600 \mathrm{mg}$ ) and paracetamol (1000 mg). ${ }^{3}$ In children, paracetamol alone is preferred (dose according to age). If the patient presents with

Figure 1. Schematic representation of progression of a lesion of dental caries and response of the dental pulp.

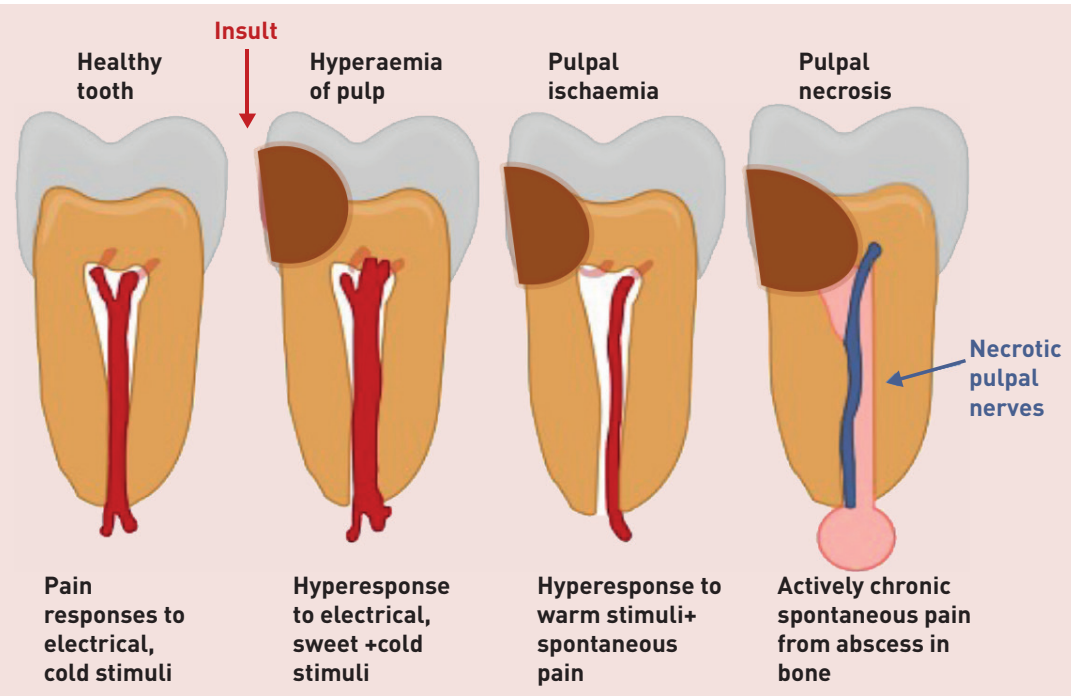

Caries - bacterial decay of enamel and dentine
Pus - made of dead white cells predominantly macrophages, due to necrotic pulp, leakage through apex ultimately causing an apical abscess 
any of the pain histories summarised above, but the pain is non-responsive to routine antiinflammatories, the clinician MUST consider the possibility of neuropathic pain. The clinician should not be tempted to prescribe antibiotics, let alone refer for surgery, as such measures are unlikely to be effective.

\section{OTHER CAUSES OF PAIN}

Dental pain can mimic many conditions and vice versa. If there is no commonly occurring form of dental pathology present, then consideration must be given to the following

Temporomandibular joints disorder (TMD). TMDs are the most common orofacial pain conditions affecting adults during periods of stress, for example, during life crises. The symptoms are preauricular pain worsened on opening wide, eating hard food, and when applying finger pressure over the joint. Dysfunctional conditions, related to nonsynchronous movements of the condylar head and the temporomandibular joint (TMJ) meniscus, include frequent clicking, crepitus, or locking of the TMJ. Diagnosis is by TMD assessment and associated tests. Management largely comprises reassurance and analgesics. Surgery is only indicated for recurrent locking.

Arthromyalgic pain, not to be confused with osteo- or rheumatoid arthritis of the TMJ, may be one or more of temporalis, masseteric, or pterygoid muscle pain. Such pain is usually related to chronic clenching or bruxism habits; it is best managed by oral splints, reassurance, and analgesics..$^{4,5}$

Headache. The symptoms mimic V2 migraine. Diagnosis and management are outlined in the recent National Institute for Health and Care Excellence guidelines. ${ }^{6}$

Maxillary sinus pain. The symptoms are pain, zygomatic tenderness, nasal congestion, and headache. Lazar et al describe investigation and treatment options.?

Otalgia. The symptoms are pain in the ear, possibly conductive hearing impairment. Diagnosis and management are directed at the identification and treatment of the causative aetiology. Acoustic neuromata, although rare, can present as toothache or TMD pain. Loss of hearing and possible facial palsy may indicate a neoplastic cause.

Trigeminal autonomic cephalalgia (TAC). The symptoms are short-lasting unilateral neuralgiform headache with conjunctival injection and tearing (SUNCT) and shortlasting unilateral neuralgiform headache attacks with cranial autonomic symptoms (SUNA) present with unilateralV2 periorbital severe pain with autonomic signs Ifacial flushing, sweating, conjunctival injection, ptosis, and nasal congestion), which distinguish TAC pain from pain of dental origin from maxillary teeth. ${ }^{8}$ Diagnosis and management depend on exact type of TAC.

Trigeminal neuralgia (TN). The symptoms are spontaneous, episodic allodynia elicited by, for example, chewing and toothbrushing. Diagnosis and management should be in accordance with European Federation of Neurological Societies (EFNS) guidelines. ${ }^{\text {? }}$

Post-traumatic neuropathy. Postoperative pain subsequent to surgical interventions, including injections, should be considered to be post-traumatic neuropathy until otherwise proven. In some cases urgent surgery is required to, for example, remove recently placed dental implants. Delay in diagnosis may result in chronic neuropathic pain, requiring medical management. ${ }^{10}$

Neoplasia. Neoplasia of the mouth is rarely painful until the late stages of the disease, or with secondary infection. If an infected tooth is near the inferior dental canal it can cause peripheral neuropathy similar to an invasive carcinoma. Investigation of a spontaneous neuropathy associated with pain must involve exclusion of neoplasia.

\section{CONCLUSION}

Dental pain is a common condition; however, patients presenting with orofacial pain may have one of a number of other conditions, but these are relatively rare. Once diagnosed, dental pain is typically best managed by operative intervention. To best help patients presenting with dental pain, in particular those with no relationship with a local dental practitioner $(<45 \%$ of the population) [British Dental Association, personal communication], medical and dental practices are encouraged to develop effective communications to facilitate the referral of patients, notably those with chronic, noncommunicable diseases such as diabetes.

If a patient with dental pain is unable to gain immediate access to dental care, including out-of-hours dental services, analgesia may help alleviate the pain. Antibiotics are rarely indicated; indeed, the prescription of antibiotics may have more negative than beneficial effects.

Finally, as with all disease, prevention should be encouraged. Whatever medical practice teams can do to promote better oral health, and thereby reduce the incidence of

\section{ADDRESS FOR CORRESPONDENCE}

\section{Nairn HF Wilson,}

King's College London Dental Institute, King's

College London, Central Office, Floor 18, Guy's

Tower, Guy's Hospital, London SE1 9RT, UK.

\section{E-mail: nairn.wilsonabtinternet.com}

acute episodes of dental pain, will be in the best interests of patients of all ages.

\section{Tara Renton,}

Professor of Oral Surgery, King's College London

Dental Institute, King's College London, London.

\section{Nairn HF Wilson,}

Professor of Dentistry, King's College London Dental Institute, King's College London, London.

\section{Provenance}

Commissioned; externally peer reviewed.

\section{DOI: 10.3399/bjgp16X684901}

\section{REFERENCES}

1. Shueb SS, Nixdorf DR, John MT, et al. What is the impact of acute and chronic orofacial pain on quality of life? J Dent 2015; 43(10): 1203-1210.

2. Woolf CJ. What is this thing called pain? J Clin Invest 2010; 120(11): 3742-3744.

3. Moore RA, Derry CJ, Derry S, et al. A conservative method of testing whether combination analgesics produce additive or synergistic effects using evidence from acute pain and migraine. Eur J Pain 2012; 16(4): 585-591.

4. Schiffman E, Ohrbach R, Truelove E, et al. Diagnostic criteria for temporomandibular disorders (DC/TMD) for clinical and research applications: recommendations of the International RDC/TMD Consortium Network and Orofacial Pain Special Interest Group. J Oral Facial Pain Headache 2014; 28(1): 6-27.

5. Manfredini D. No significant differences between conservative interventions and surgical interventions for TMJ disc displacement without reduction. Evid Based Dent 2014; 15(3): 90-91.

6. National Institute for Health and Care Excellence. Headaches in over 12s: diagnosis and management. CG150. London: NICE, 2012. https://uww.nice.org.uk/guidance/cg150 (accessed 5 Apr 2016).

7. Lazar ML, Greenlee RG Jr, Naarden AL. Facial pain of neurologic origin mimicking oral pathologic conditions: some current concepts and treatment. JAm Dent Assoc 1980; 100(6): 884-888.

8. May A, Leone M, Afra J, et al. EFNS guidelines on the treatment of cluster headache and other trigeminal-autonomic cephalalgias. Eur J Neurol 2006; 13(10): 1066-1077.

9. Cruccu G, Gronseth G, Alksne J, et al. AANEFNS guidelines on trigeminal neuralgia management. Eur J Neurol 2008, 15(10): 1013-1028.

10. National Institute for Health and Care Excellence. Neuropathic pain in adults: pharmacological management in non-specialist settings. CG173. London: NICE, 2013. https://mww.nice.org.uk/ guidance/cg173 (accessed 5 Apr 2016). 